\title{
L'ouïe en tant que stratégie de construction du récit de soi chez Louis-Ferdinand Céline
}

\section{Abstract (En/Fr)}

Loredana Trovato $^{1}$

This article analyzes the role of hearing Louis-Ferdinand Celine's novels and its relationship with the construction processes of self-narration. Since Journey to the End of Night was first published, hearing has always been the sense most used by the author, appearing both in the dynamic interaction with the outside world (in terms of conflict and/or symbiotic relationship with the reader/narratee) and in the mechanisms of internal 'benchmark' for experienced biographical data ('oto-biography') which become 'oto-memory' and eventually 'oto-fiction'. The auditory memory, haunting his head through the noise and the endless chatter, forces him to write, that is to say to leave reality to go to the fiction of reality, in a kind of act of maieutics. Through the analysis of the auditory leitmotifs in Celine's work, we shall try to determine the basic features of his poetics and the uniqueness of a style that he rightly called himself 'little music'.

Cet article vise à analyser le rôle de l'ouïe dans l'œuvre romanesque de Louis-Ferdinand Céline et ses rapports avec les stratégies de construction du récit de soi. Dès la parution de Voyage au bout de la nuit, l'ouïe a toujours été un des sens les plus utilisés par l'auteur, intervenant dans l'interaction dynamique avec l'extérieur (en termes de conflit et/ou de relation symbiotique avec le lecteur/narrataire), mais aussi dans les mécanismes de 'repère' intérieur des données du vécu biographique ('oto-biographie') qui deviennent 'oto-mémoire' et, enfin, 'oto-fiction'. Comme une sorte d'acte maïeutique, le souvenir auditif qui hante sa tête par ses bruits et ses jacassements sans fin, lui impose l'écriture, c'est-à-dire le passage du réel à la fiction du réel. À travers l'analyse des leitmotivs auditifs de l'œuvre célinienne, on essayera ainsi de déterminer les traits essentiels de sa poétique et l'unicité d'un style qu'il appelle - à juste titre - 'petite musique'.

\section{Les mécanismes auditifs dans l'œuvre célinienne}

Le titre de cette communication a la 'petite' présomption de s'entendre en tant qu'une proposition (assez) catégorique: l'ouïe est l'une des stratégies les plus importantes utilisées par Céline dans la construction du récit et, en particulier, du récit de soi. Elle est une des composantes

\footnotetext{
${ }^{1}$ Université Kore d'Enna.
} 
essentielles de son alchimie créatrice et intervient dans la structuration du logos à travers un procédé et mécanique de transformation du son en écriture (muette) sur la feuille vierge, et de recomposition psychanalytique de l'enfer des souvenirs dans la fiction romanesque. Ce procès constitue le modèle de base de son œuvre car il implique la dimension proprement linguistique et celle générique ou thématique. Bref, toute l'œuvre dans sa globalité et unicité.

En ce qui concerne la dimension linguistique, c'est Céline lui-même qui définit son écriture comme une 'petite musique', un 'style émotif' et musical, dans le livre qui révèle la substance de sa poétique, Entretiens avec le Professeur Y (1955). Il reconnaît aussi sa dette envers le 'bruit dans la tête' qui, comme une sorte de moteur créateur, ne lui permet pas de se reposer pendant les nuits où son oreille mutilée l'amène par les chemins des souvenirs.

L'ouie est ce démon socratique qui est censé le pousser vers la libération maïeutique des inquiétudes et des cauchemars de son âme. Voilà pourquoi c'est le sens qu'on perçoit le mieux dans sa production et qui se place au point d'origine ' 0 ' dans la composition paradigmatique (axe vertical du domaine linguistique) et syntagmatique (axe horizontal du domaine de l'architecture fictionnelle). Les deux axes peuvent se développer à l'infini à partir de la même expérience sensorielle (l'expérience auditive) jusqu'à déterminer de façon qualitative les 'extrêmes' de la productivité du texte célinien.

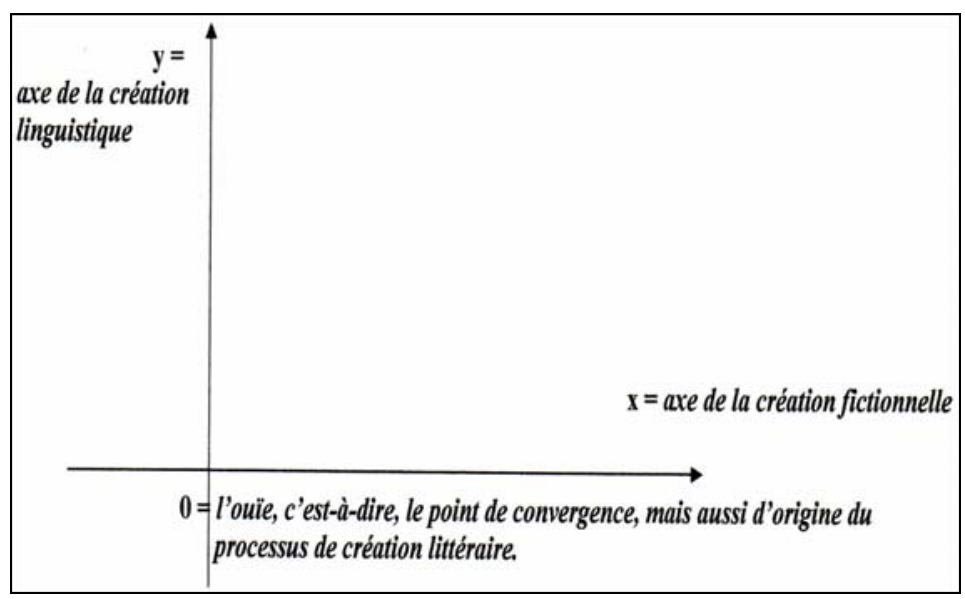


À partir de ces quelques considérations, on peut affirmer que l'écriture célinienne relève du 'pseudo-oto-biographique' ou de l' oto-fiction', le 'o' à la place de la diphtongue 'au' soulignant le préfixe 'oto' qui indique l'oreille, la perception auditive. De plus, on aimerait aussi utiliser le terme derridien d' oto-biographie' pour mettre en relief le fait que les données biographiques céliniennes les plus importantes sont fortement liées aux bruits, aux sons, à la capacité et/ou nécessité d'entendre, d'écouter, d'oreiller, d'ouïr... À ce propos, il faut rappeler que l'événement marquant la vie et l'œuvre de Céline est la guerre, capharnaüm de bruits dissociés, (dis)harmonie fluctuante et décomposée de sons: cette guerre qui l'a mutilé pendant les bombardements (explosion hyperbolique de bruits) sur la ligne des Flandres, dont le souvenir le hante la nuit lorsqu'il entend encore les clameurs de la bataille dans sa tête.

Un autre schéma peut rendre compte alors du passage de l'oto-biographie à l'oto-fiction par ce qu'on pourrait appeler 'oto-mémoire', ou mémoire auditive, c'est-à-dire la mémoire déclenchée par le tohu-bohu auditif, mécanisme qui rappelle - certes! - la célèbre madeleine proustienne.

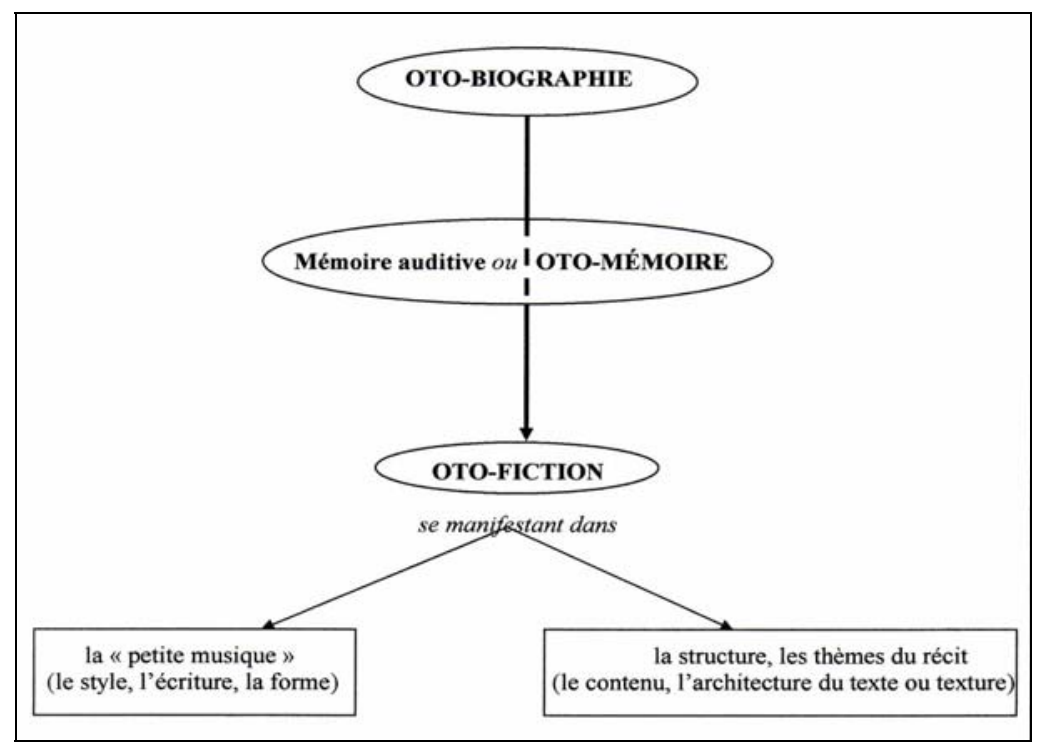


L'oto-mémoire constitue une sorte de filtre et/ou de trait d'union dans le passage de l'otobiographie à l'oto-fiction: comme il advient souvent dans la communication orale où le message de départ peut être altéré par les interférences du canal, le vécu biographique se métamorphose en fiction du réel lorsqu'il est altéré par les interférences ininterrompues de la mémoire auditive qui, dans la tête, cherche à apaiser la douleur physique et psychique de l'écrivain.

\section{Des données oto-biographiques à l'oto-mémoire}

Ce qui semble évident, mais qu'il faut souligner, c'est la coïncidence sur le plan de l'expérience - le plan factuel - entre l'oto-biographie et l'oto-mémoire. La différence réside par contre sur le plan temporel: si, dans l'oto-biographie, le temps est (dé)fini, délimité au seul moment où l'action se déroule et donc, conclu, circonscrit, fermé, dans l'oto-mémoire, le temps du souvenir peut se répéter à l'infini et revenir à l'occurrence. Tout au long de ce procès de répétition, le souvenir peut se modifier, c'est-à-dire qu'une des composantes de ce souvenir peut échapper au mouvement circulaire et suivre une autre direction pour devenir oto-fiction, ce qu'on a essayé de mettre en évidence dans le tableau suivant:

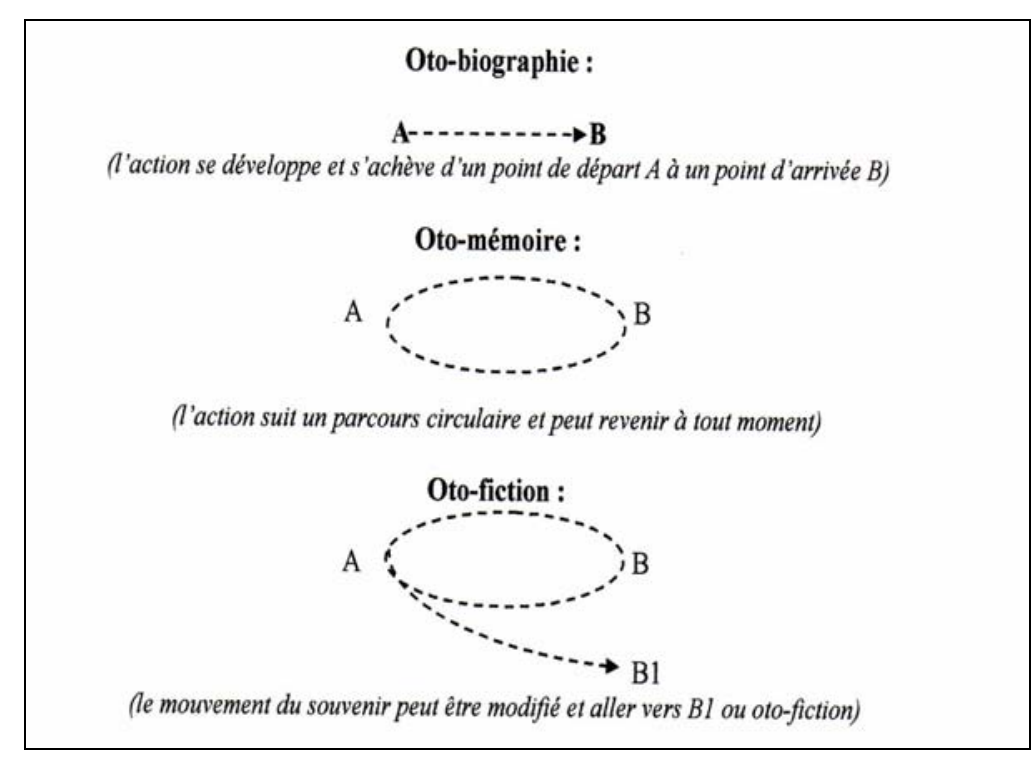


Pour mieux focaliser l'attention sur le récit de soi, on ne proposera pas une analyse des éléments oto-biographiques, mais on en fera une liste assez brève afin de créer le lien nécessaire avec les données de l'oto-mémoire en transition vers l'oto-fiction. D'abord, les éléments otobiographiques céliniens principaux sont trois et constituent les leitmotivs de sa production romanesque, ainsi que de sa correspondance privée: en particulier, on peut repérer la présence constante $a$ ) de la 'voix' de la mère sous forme de conseils et avertissements; $b$ ) des 'voix' multiples de la guerre; $c$ ) des (dis)harmonies imitatives, des bruits des objets et des machineries, des sonorités ou dissonances humaines de la ville (Paris, ou bien Londres). Ces trois points correspondent aux parties fondamentales de sa vie, celles qu'on retrouve - 'fictionnalisées' - en tant que sujets et actants de ses romans, mais qui apparaissent, premièrement, dans les entretiens et le vaste corpus épistolaire comme des manifestations presque obsessives et paranoïaques de la mémoire auditive.

Jean-Claude Renard dans Céline, les livres de la mère (2004) affirme que, chez l'auteur, la fonction de la mère est nécessaire au développement du récit et stratégique au niveau de l'écriture puisqu'elle représente son verbe et sa verve: «En filigrane ou dans un premier rôle, suggérée, invoquée, racontée, la mère du narrateur, tantôt sorcière, tantôt bonne fée, envahissante ou inaccessible, pathétique ou sublime, déambule ainsi tout au long de l'œuvre. Outre une présence constante dans la fiction, il n'est pas de correspondance dans laquelle elle n'apparait pas, il est peu d'entretiens accordés par l'auteur dans lesquels elle n'est pas évoquée» ${ }^{2}$.

Cette présence est tellement stable au niveau du souvenir et, surtout, du souvenir auditif qu'il serait presque impossible d'en citer toutes les occurrences textuelles. Céline aime bien rapporter les mots de la mère à travers le discours direct ou indirect: lorsqu'il rappelle son métier de commerçante de dentelles au Passage Choiseul avec ses impératifs, sa complaisance envers le client et sa soumission presque inconditionnelle, il utilise le discours direct afin de mieux souligner le fatalisme de sa pensée:

«Ma mère me disait toujours: 'Petit malheureux, si tu n'avais pas les gens riches, [...] s'il n'y avait pas les gens riches, nous n'aurions rien à manger. Ben, les gens riches ont des responsabilités...' Ma mère révérait les gens riches, n'est-ce pas» ${ }^{3}$.

\footnotetext{
${ }^{2}$ Jean-Claude RENARD, Céline, les livres de la mère, Paris, Buchet/Chastel, 2004, p. 14.

${ }^{3}$ Entretien avec Jean GUÉNOT et Jacques DARRIBEHAUDE, in Cahiers Céline, 2, Céline et l'actualité littéraire (1957-1961), textes réunis et présentés par Jean-Pierre DAUPHIN et Henri GODARD, Paris, Gallimard, 1976, p. 163.
} 
L'ascoltare, il sentito dire, la phonè nei racconti di sé

Le même concept revient sous forme de discours indirect:

«Ma mère me faisait de la morale elle-même. Elle me faisait toujours remarquer que la cliente était un objet sacré, qu'elle avait des responsabilités que je ne soupçonnais pas et que c'est grâce à elle que nous vivions et que je ne pouvais pas imaginer même le sacrifice et la vertu des gens riches» ${ }^{4}$.

Si le discours de type direct relève d'un mécanisme de reproduction directe du souvenir auditif et, par conséquent, de reproduction assez fidèle du vécu biographique, le discours de type indirect présuppose par contre une médiation, une réélaboration de la perception auditive qui devient, enfin, oto-fiction, c'est-à-dire 'fictionnalisation' de la réalité écoutée, entendue, perçue.

Même le thème de la guerre avec sa sarabande de bruits, fracas, sonorités dispersées ou concentrées se répète d'une page à l'autre tout au long de sa production. Ce sujet se manifeste souvent de deux façons: l'une - directe - à travers la mémoire auditive de la bataille et des bombardements, l'autre - indirecte - à travers l'allusion à sa mutilation physique (le bras paralysé et le 'bourdonnement des oreilles'). C'est ce bourdonnement, ce bruit dans les oreilles qui l'entraine à l'écriture et à la 'petite musique' de son style:

«Mais la nuit que faire quand on ne dort pas? J'écoute dans mon oreille la folle du logis. Elle m'en raconte, allez, depuis six ans que j'écris ce livre» ${ }^{5}$.

Céline revient plusieurs fois à ce sujet dans les entretiens et la correspondance qui précèdent sa fuite au Danemark; toutefois, à partir de la période de l'emprisonnement, si dans le corpus épistolaire il semble réclamer du silence et de la solitude, c'est dans les romans (et, principalement, dans Féerie pour une autre fois) que la 'nuisance sonore' explose bruyante et harmonique et «l'ouïe prend souvent une dimension fantastique» ${ }^{6}$. C'est alors qu'elle crée, définit, harmonise les images; qu'elle compose sa féerie de personnes, objets, éléments abstraits; qu'elle métamorphose, métaphorise et personnifie Paris ou bien Londres dans deux êtres

\footnotetext{
${ }_{5}^{4}$ Entretien avec Pierre DUMAYET, op. cit., p. 63.

${ }^{5}$ Interview de Merry BROMBERGER à Louis-Ferdinand Céline parue sur l'Intransigeant en 1933 après la publication de Voyage au bout de la nuit. In Cahiers Céline 1 - Céline et l'actualité littéraire 1932-1957, textes réunis et présentés par Jean-Pierre DAUPHIN et Henri GODARD, Paris, Gallimard-NRF, 1976, p. 30.

${ }^{6}$ Sonia ANTON, Céline épistolier. Écriture épistolaire et écriture littéraire, Paris, Éditions Kimé, 2006, p. 251.
} 
amorphes et contingents, qui évoluent en fonction de la variation des tons, des timbres et des sonorités multiples de leur essence profonde et vitale.

\section{De l'oto-mémoire à l'oto-fiction: des 'paysages' de l'esprit aux 'passages' de la fiction}

C'est dans les romans que Céline offre les exemples les plus parfaits d'oto-fiction: ils sont, en effet, une «symphonie littéraire» ${ }^{7}$ ou, comme le dit Henri Godard, «la mise en forme d'une matière sonore» ${ }^{8}$. Dès le Voyage au bout de la nuit (1932) - œuvre partiellement auto- et otofictive - l'auteur manifeste une attention vive à la matière auditive, recomposant et 'fictionnalisant' ses souvenirs à travers l'ouïe. Ainsi, après la célèbre expression «ça a débuté comme ça», Céline exploite soudain sa prédilection pour l'ouïe en l'utilisant comme un sésame qui ouvre les portes de son récit:

«Moi, j'avais rien dit. Rien. C'est Arthur Ganate qui m'a fait parler. [...] Il veut me parler. Je l'écoute» ${ }^{9}$.

Voyage au bout de la nuit met en scène les aventures de Ferdinand Bardamu à la recherche de son Graal à travers de multiples expériences parmi lesquelles, la guerre. L'écho de cette voltairienne «boucherie hérö̈que» ${ }^{10}$ est l'élément qui pousse Ferdinand à s'engager, au point qu'il admet sa fascination pour le régiment, «le colonel et sa musique» ${ }^{11}$. La voix du colonel Des Entrayes avec son exhortation à la bataille reviendra souvent dans les autres romans (en premier lieu, dans Guignol's band I et II) sous forme de mémoire auditive 'fictionnalisée', d'avertissement moral ou de leçon éthique pour le protagoniste.

Mort à crédit (1936), sorte d'anti-bildungsroman, présente le chemin d'anti-formation à la vie de Ferdinand, héros dont le prénom rappelle bien, encore une fois, celui de Céline. L'auteur y rassemble tous ses souvenirs d'adolescence à travers la mémoire auditive qui lui

\footnotetext{
${ }^{7}$ Céline même, dans une lettre à l'éditeur Gallimard, utilise cette expression pour résumer le style du manuscrit soumis. In Louis-Ferdinand CÉLINE Lettres à la N.R.F. 1931-1961, Paris, Gallimard, 1961, p. 14.

${ }^{8}$ Henri GODARD, Voyage au bout de la nuit de L.-F. Céline, Paris, Gallimard-Foliothèque, 1991, p. 140.

${ }^{9}$ Louis-Ferdinand CÉLINE, Voyage au bout de la nuit, in Romans I, édition présentée, établie et annotée par Henri GODARD, Paris, Gallimard, coll. 'Bibliothèque de la Pléiade', 1981, p. 7.

${ }^{10}$ VOLTAIRE, Candide ou l'optimisme, Paris, Larousse, 1990, p. 37.

${ }^{11}$ L.-F. CÉLINE, Voyage au bout de la nuit, op. cit., p. 10.
} 
suggère les 'voix' de la famille, ainsi que les multiples sonorités et/ou dissonances de la ville de Paris, véritable corps sensible et tohu-bohu de bruits. Et pourtant, c'est la mère qui domine le roman, si bien que maints critiques l'ont défini comme le roman maternel ou le roman le plus familial de Céline. Sa voix retentit d'une page à l'autre et le fils en rappelle la chaleur ou l'aigreur, la colère ou le sourire, le ton paisible ou l'âpreté du reproche:

«Je l'ai vue chialer chaque soir ou presque, ma mère, à cause de son ouvrière et des dentelles qui revenaient pas» ${ }^{12}$; «Mme Pinaise se rapproche. Ils s'amusent à m'agacer, tous les deux. Ma mère parle toujours. Ses boniments servent à rien» ${ }^{13}$. «Ma mère escaladait sans cesse, à cloche-pied. Ta! pa! tam! Ta! pa! tam! Elle se retenait à la rampe. Mon père ça le crispait de l'entendre» ${ }^{14}$.

À côté des souvenirs de famille, Céline propose une peinture vivante du Passage Choiseul et de la ville de Paris; toutefois, il ne manque jamais de rapprocher le tableau visuel de la représentation des sonorités multiples et variées. Il raconte alors le Paris du chaos humain et déshumanisé fin de siècle, de la frénésie de l'époque du progrès industriel dont le résultat le plus magnifique avait été l'Exposition universelle de 1900, des quartiers coloriés, multiformes et vifs, des gens chahutant dans les rues:

«Voici le train qui vrombit, c'est un tonnerre, on dirait qu'il arrache tout. Les voyageurs se trémoussent, se décarcassent, chargent en ouragan les portières» ${ }^{15} ;$ «On s'est retrouvés ahuris dans la Galerie des Machines, une vraie catastrophe en suspens dans une cathédrale transparente en petites verrières jusqu'au ciel. Tellement le boucan était immense que mon père on l'entendait plus, et pourtant il s'égosillait. La vapeur giclait, bondissait par tous les bords» ${ }^{16}$.

Des descriptions semblables se multiplient dans le texte pour constituer une vaste harmonie imitative qui, sollicitant l'oto-mémoire, définit et compose l'oto-fiction célinienne. Cette harmonie devient cependant disharmonie dans Guignol's band I et $I I$, où le fantôme de la guerre, les conflits d'une bande de démunis échappés à l'horreur des champs de bataille français et ré-

\footnotetext{
${ }^{12}$ L.-F. CÉLINE, Mort à crédit, op. cit., p. 547.

${ }^{13}$ Ivi, p. 562.

${ }^{14}$ Ivi, p. 560.

${ }^{15}$ Ivi, p. 546.

${ }^{16}$ Ivi, p. 579.
} 
fugiés à Londres composent des démons inquiets qui s'agitent et bourdonnent dans la tête et les oreilles de l'auteur.

Guignol's band I s'ouvre en effet sur le récit fictionnel d'une oto-mémoire, c'est-à-dire sur le souvenir des bombardements de la ville d'Orléans en 1940, où le réalisme de la représentation est accentué par la présence de nombreuses interjections onomatopéiques qui évoquent le bruit de la bataille et des explosions:

«Braoum! Vraoum!... C'est le grand décombre!... Toute la rue qui s'effondre au bord de l'eau! [...] Le Fritz mitraille épouvantable, s'apporte là-haut du fond des cieux! La vache! De son brezinzin il nous rase! Il nous asperge des plus hautes cimes, il nous enveloppe, il nous vrombit!» ${ }^{17}$.

Dans Guignol's band, le titre aussi exprime la prédominance des mécanismes de l'oralité, de l'ouïe en tant que moyen d'interaction et/ou de rupture avec le monde, car le mot 'band' peut bien rappeler un groupe musical. De même, le protagoniste Ferdinand communique ou refuse de communiquer avec le contexte et ses personnages à travers sa faculté d'entendre, de saisir l'essence du réel par un acte de perception auditive:

«Je les [Prospero et Cascade] écoute jacasser... Ça me concernait pas toujours... J'aurais pu dire mon petit mot! Mais salut! je ferme ma gueule!... Chacun pour soi l'expérience!»» ${ }^{18}$.

Les bruits sont des éléments essentiels dans la struggle for life qu'engage Ferdinand pendant son expérience londonienne: il frémit, palpite, ressent de l'horreur et du dégoût lorsqu'il entend même de loin le «Poëp! Poëp!» de Nelson qui résonne dans l'air épais de la ville ou le «bruit d'os» de la «carcasse d'épouvantail» de Mille-Pattes:

«Il crisse c'est son rire... ses mâchoires crispent râpent... entrechoquent... une vieille pendule dans sa bouche... c'est sa rigolade... il est d'accord, il jubile!... il fait son gras bruit d'os exprès $[\ldots]$ il est complet il est inouï...» ${ }^{19}$.

${ }^{17}$ L.-F. CÉLINE, Guignol's band I, in Romans III, édition présentée, établie et annotée par Henri GODARD, Paris, Gallimard, coll. «Bibliothèque de la Pléiade», 1988, pp. 87-88.

${ }^{18}$ Ivi, p. 163.

${ }^{19}$ L.-F. CÉLINE, Guignol's band II, op. cit., p. 472. 
Dans ses promenades londoniennes, il est toujours aimanté par les bruits et les sonorités de la ville avec ses docks mirifiques, les enfants qui beuglent joyeusement dans les rues, les tramways qui transportent de milliers de personnes avec leurs dissonances multiples:

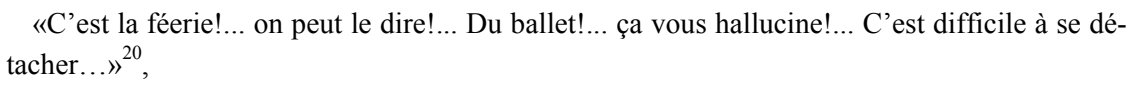

s'écrie le narrateur face au spectacle de la ville bruyante et mouvementée.

À partir de Guignol's band I, la féerie devient l'une des notions les plus importantes de la poétique célinienne, car elle résume au fond l'amour de Céline pour la musique, l'harmonie, l'émotion, la légèreté, les (atmo)sphères célestes pour s'exprimer le mieux dans Féerie pour une autre fois I (1952) et II (1954).

Dans Féerie pour une autre fois, la voix de la mère est, par exemple, un canal physique tangible et fort efficace du passage de l'oto-mémoire à l'oto-fiction, car elle se métamorphose (graphiquement et phonétiquement) en musique, comme on peut l'observer de l'image-citation suivante $^{21}$ :

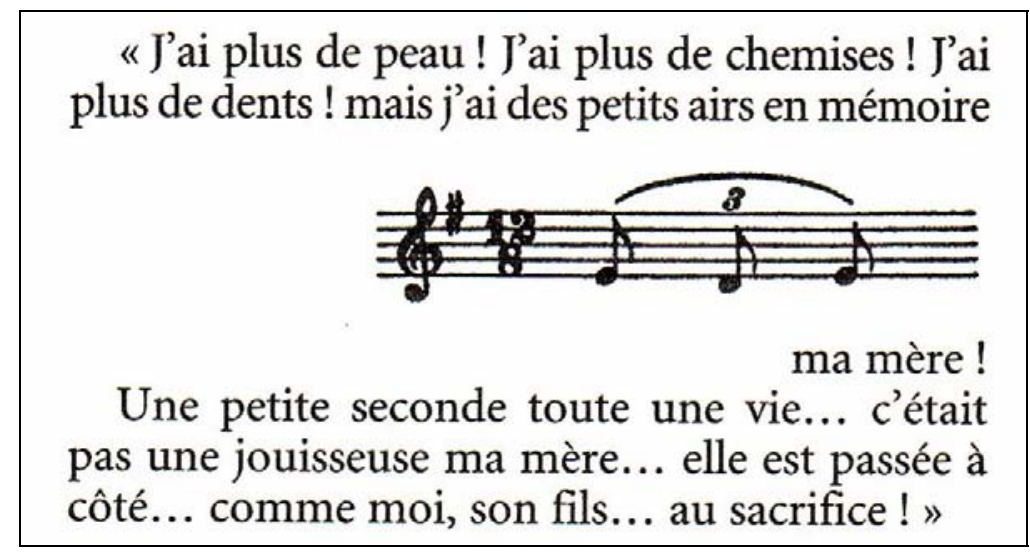

\footnotetext{
${ }^{20}$ L.-F. CELINE, Guignol's band I, op. cit., p. 179.

${ }^{21}$ L.-F. CELINE, Féerie pour une autre fois $I$, in Romans $I V$, édition présentée, établie et annotée par Henri GODARD, Paris, Gallimard, coll. 'Bibliothèque de la Pléiade', 1981, pp. 161-162.
} 
À côté du thème de la mère, Féerie pour une autre fois I est foncièrement le livre de l'otomémoire qui devient oto-fiction: Céline relate la guerre et la période de l'entre-deux-guerres à travers un ton mélancolique, musical, lyrique et, si l'on veut, idyllique. Le souvenir, c'est la chanson, la musique, l'harmonie de la danse qui déclenchent un éclatement magnifique de bribes de la mémoire:

Vous $^{22}$ m'aurez pas la rigolade! Je vogue! Je vogue! et la chanson!... Je vous entends rire! les notes, vous voudriez? les notes? Je les ai aussi dans la tête! Je vais vous les écrire, transcrire!... Vous vous chercherez un piano... prenez pas celui à Jules ${ }^{23}$ ! Allez pas chez Jules!... il est faux le piano à Jules! faux chez Jules!... la vraie note que vous jouerez! la vraie note! et puis une autre! et la chanson!

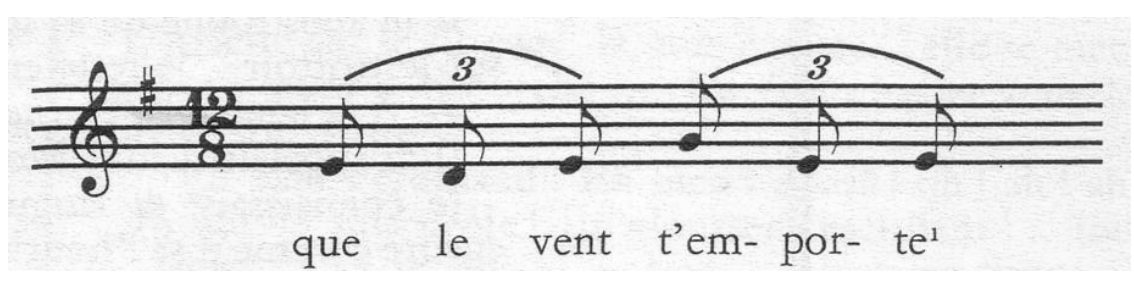

Vous vous trouverez bien un piano! avec un doigt vous vous jouerez!... Ils sont pas tous morts les pianos! C'est des bruits qui existent encore! le mi! le ré!... vous avez pas besoin de bugle! en fa dièse! Y a des bruits qui sont finis... mais un piano! un doigt! un autre! y a des bruits qui existent plus!... mais le piano: fa dièse et sol! $!^{24}$

Dans la citation suivante, le souvenir des personnes qu'il a rencontrées avant de s'enfuir de la France est lié à la musique et à la chanson:

«Moi je le connais le filon à Jules! J’y ai vu tante Estrême, Ciboire et le petit Léo!... J'ai vu Clémence!... Je me suis sauvé avec les notes» ${ }^{25}$.

\footnotetext{
22 'Vous' est le narrataire, le lecteur implicite qui devient, à partir de Féerie pour une autre fois, l'interlocuteur principal du narrateur.

${ }^{23}$ Le personnage de Jules a été modelé sur l'image de l'écrivain et ami de Céline, Gen Paul.

${ }^{24}$ L.-F. CÉLINE, Féerie pour une autre fois I, op. cit., pp. 158-159.

${ }^{25}$ Ivi, p. 167.
} 
L'ascoltare, il sentito dire, la phonè nei racconti di sé

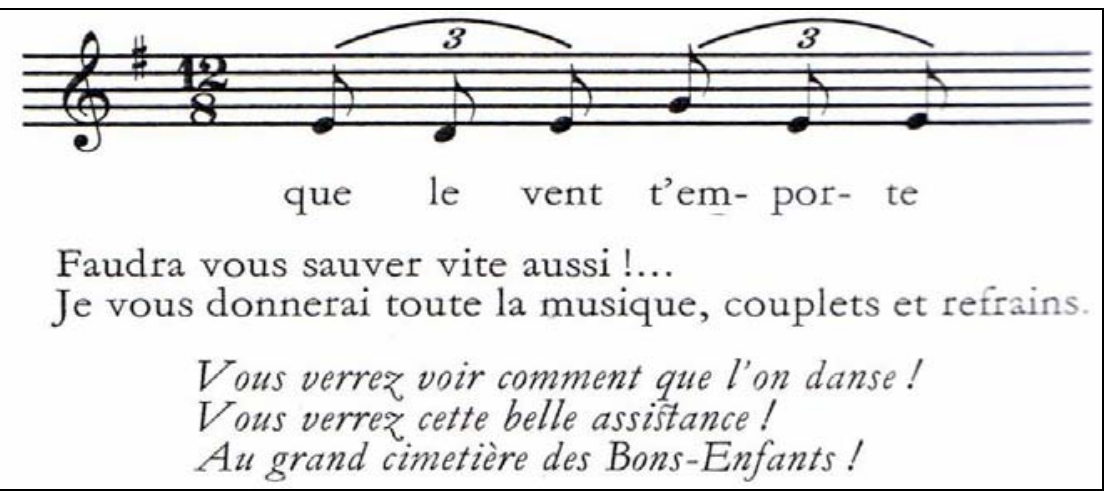

Féerie pour une autre fois I s'achève sur l'image du narrateur qui, enfermé dans le cachot danois et accablé par la série continue de ses mésaventures, associe les sonorités de l'extérieur avec le souvenir des amis et de sa femme:

«Je souffre de partout entendu!... J'entends hululer les chouettes! J'entends les sirènes du port! mais stoïque, harmonieux, aimable... Je sais des trucs!... Je remémore, y a du condé! Je pense à Jules, je pense à Lili...» ${ }^{26}$.

Dans les œuvres postérieures, Féerie pour une autre fois II et la Trilogie allemande (D’Un château l'autre, Nord, Rigodon) l'oto-mémoire première est la guerre qui devient oto-fiction à travers une écriture à la phrase plus fragmentaire et fragmentée, au rythme ininterrompu et musical, recourant plus souvent aux interjections onomatopéiques qu'auparavant. Il est important de souligner, à ce propos, que l'action de Féerie II est structurée à partir du souvenir d'une seule journée de l'auteur qui, réfugié dans son appartement de Montmartre, voit et entend bombarder la ville. Voici, à ce propos, l'incipit:

«Raconter tout ça après... c'est vite dit!... c'est vite dit!... On a tout de même l'écho encore... brroum!... la tronche vous oscille... même sept ans passés... le trognon!... le temps n'est rien, mais les souvenirs! [...] Nom de brise! J'en ai plein la tête!» ${ }^{27}$; «Je ne sais pas si

\footnotetext{
${ }^{26}$ Ivi, p. 175.

${ }^{27}$ L.-F. CÉLINE, Féerie pour une autre fois II, op. cit., p. 179.
} 
L. Trovato: L'ouïe en tant que stratégie de construction de soi chez Céline

c'est l'effet des chocs mais ils me semblent là tout autour encore plus abrutis que moi-même mes amis porteurs... ils savent que hein Ferdinand?... hein?... hein?... je les entends qu'à peine... hein?... hein? hein? J'ai des bruits personnels un peu... je vous ai dit... et puis des bombes!» ${ }^{28}$.

Ces quelques exemples témoignent de la fonction significative et, finalement, paradigmatique de la composante auditive dans les textes céliniens qui, loin de concrétiser seulement une stratégie de construction du récit ou de constituer une épreuve de l'authenticité du raconté, se manifeste aussi en tant qu'élément segmental et suprasegmental. Autrement dit, elle est porteuse, à la fois de l'unité significative et de l'unité signifiante de la narration et détermine l'architecture globale de la production de l'auteur tout au long de $\mathrm{sa} \mathrm{vie}^{29}$.

\footnotetext{
${ }^{28}$ Ivi, p. 181.

${ }^{29}$ On consultera encore à propos du sujet traité: Anne HENRY, «Réflexions sur la 'petite musique' de Céline», in Voix et création au XX $X^{e}$ siècle, Paris, Honoré Champion, 1996.
} 